\title{
Application of ProteinChip Array Profiling in Serum Biomarker Discovery for Patients Suffering From Severe Acute Respiratory Syndrome
}

\author{
Timothy T. C. Yip, William C. S. Cho, Wai Wai Cheng, \\ Johnny W. M. Chan, Victor W. S. Ma, Tai-Tung Yip, \\ Christine N. B. Lau Yip, Roger K. C. Ngan, and Stephen C. K. Law
}

\begin{abstract}
Summary
A new strain of coronavirus has caused an outbreak of severe acute respiratory syndrome (SARS) from 2002 to 2003 resulting in 774 deaths worldwide. By protein chip array profiling technology, a number of serum biomarkers that might be useful in monitoring the clinical course of SARS patients were identified. This book chapter describes how the protein chip array profiling was carried out for this study. Briefly, SARS patients'serum samples were first fractionated in Q Ceramic HyperD ion exchange sorbent beads by buffers at different $\mathrm{pH}$. Serum protein fractions thus obtained were then bound onto a copper (II) immobilized metal affinity capture (IMAC30 Cu [II]) ProteinChip ${ }^{\circledR}$ Array or a weak cation-exchange (CM10) ProteinChip Array. After washing and addition of sinapinic acid, the chips were read in a Protein Biological System (PBS) IIc mass spectrometer. Ions were generated by laser shots and flied in a time of flight mode to the ion detector according to their mass over charge $(\mathrm{m} / \mathrm{z})$ ratio. The serum profiling spectra in SARS patients were acquired, baseline subtracted and analyzed in parallel with those from the control subjects by Ciphergen ProteinChip Software 3.0.2 with their peak intensities compared by a nonparametric two sample Mann-Whitney- $U$ test. More than twelve peaks were differentially expressed in SARS patients with one at $\mathrm{m} / \mathrm{z}$ of 11,695 (later identified to be serum amyloid A protein), which had increase in peak intensity correlating with the extent of SARS-coronavirus induced pneumonia as defined by a serial chest X-ray opacity score. The remaining biomarkers could also be useful in the study of other clinical parameters in SARS patients.
\end{abstract}

Key Words: Pneumonia; ProteinChip array profiling; SARS-Coronavirus (CoV); serum amyloid A protein (SAA); severe acute respiratory syndrome (SARS); surface-enhanced laser desorption/ionization time of flight mass spectrometry (SELDI-TOF-MS). 


\section{Introduction}

A new strain of coronavirus $(\mathrm{CoV})$ caused a pandemic outbreak of severe acute respiratory syndrome (SARS) in China, Hong Kong, Singapore, Toronto, and Taiwan from 2002 to 2003, resulting in 8098 individuals being infected and 774 deaths (1). As compared to other upper viral respiratory infections (2), SARS-CoV can rapidly induce pneumonia. The accompanying adult respiratory distress syndrome can quickly progress resulting in rapid death of the patients $(3,4)$. The most commonly used laboratory diagnostic tests for SARS-CoV-infection are reverse transcription (RT)-PCR and anti-SARS-CoV antibody serological tests (5-7), but their roles in monitoring the extent of pneumonia are rather limited. Although, serial chest radiography is helpful for monitoring the progress of disease $(\mathbf{8 , 9})$, it is also limited by its subjectivity and variability in the interpretation of the imaging results and occasionally suboptimal quality of portable films taken in isolation ward to avoid the spread of the disease. In a recent article, the discovery of 12 upor downregulated serum biomarkers were reported in SARS patients by a novel protein chip array profiling approach (10-14) with one biomarker appears to be useful in monitoring the extent of pneumonia (15). In this chapter, how this protein chip array profiling technique is carried out is described. The technique involves four steps with the first step being serum fractionation in ceramic ion exchange sorbent beads. This step separates serum proteins into several fractions aiming at reducing their complexity before protein chip profiling is performed. The second step involves the binding of serum protein biomarkers onto protein chip array surfaces. The third step is desorption of bound serum biomarkers by laser shots in the mass spectrometer generating a time of flight spectrum for each sample. The fourth step concerns with data acquisition, data processing and statistical analyses of the spectra in SARS patients in comparison with the control groups. These procedures will be described in full details in the following chapter and summarized in the flow chart diagram as in Fig. 1.

\section{Materials}

\subsection{SARS Patients}

1. 28 Patients with confirmed SARS-CoV infection managed in the Department of Medicine, Queen Elizabeth Hospital, Hong Kong.

2. $87 \%$ of the patients have abnormal chest radiographs on admission.

3. $13 \%$ of the patients present early thus requiring high-resolution thoracic computer tomography scanning to confirm the presence of pulmonary involvement.

\subsection{Serum Samples}

1. 44 Serum samples from 24 SARS patients for differential mapping of SARS associated biomarkers. 


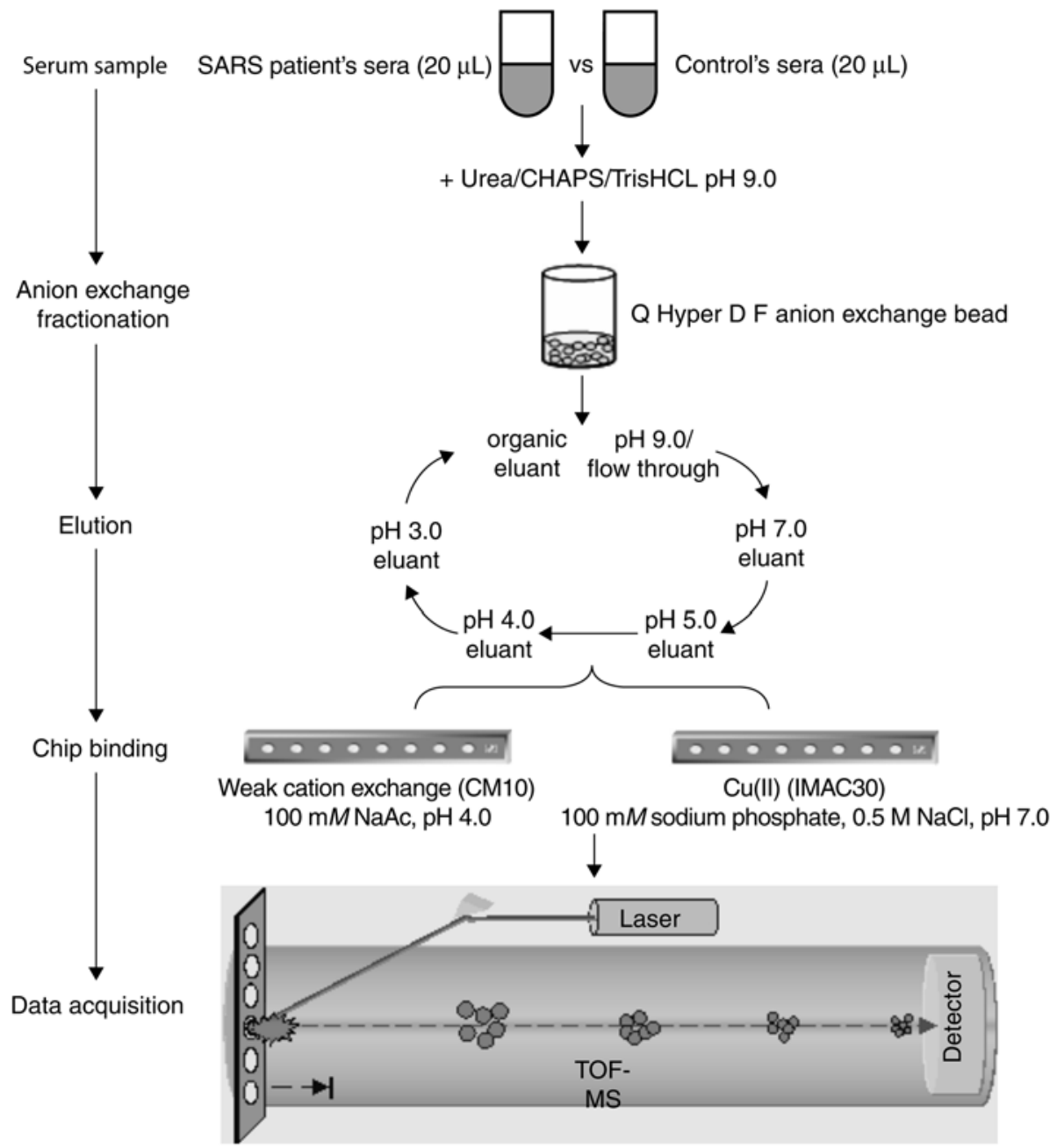

Protein biology system (PBS) II SELDI-TOF mass spectrometer

Fig. 1. Sample fractionation, chip binding, and reading in protein chip array profiling technique. Serum samples from SARS patients and various control groups were fractionated in Q Ceramic Hyper D F anion exchange sorbent beads. The eluted fractions were in turn spotted onto two types of protein chips, namely, copper IMAC30 $\mathrm{Cu}$ (II) ProteinChip array and weak cation-exchange (CM10) ProteinChip array. Each sample bound protein chip was in turn inserted into a Protein Biology System II SELDI-TOF-mass spectrometer and the protein biomarkers were ionized and desorbed by laser beam. The ions flied to the ion detector generating a biomarker peak intensity spectrum according to their mass over charge values $(\mathrm{m} / \mathrm{z})$. 
2. 45 Serial serum samples from four additional SARS patients with comprehensive clinical follow-up for longitudinal correlation with clinical manifestation.

3. 10 Sera from 10 apparently healthy individuals (from a familial cancer screening clinic) as normal controls.

4. 72 Sera from 51 patients suffering from other viral or bacterial infections (influenza A virus $[n=12]$, influenza $\mathrm{B}$ virus $[n=8]$, respiratory adenovirus $[n=12]$, respiratory syncytial virus $[n=10]$, hepatitis B virus $[n=10]$, Mycobacterium tuberculosis $[n=10]$, other bacteria $[n=10])$ as control patients for comparison with SARS patients.

\subsection{Ion Exchange Fractionation of Serum}

1. BioSepra Q Ceramic Hyper D F ion exchange sorbent beads (Ciphergen Biosystems Incorporation, Fremont, $\mathrm{CA}$ ).

2. 96-Well sample plates (Nalge Nunc International, Rochester, NY).

3. $96-W e l l$ Silent Screen filter plates with Loprodyne membrane filter $(0.45 \mu \mathrm{m}$ pore size; Nalge Nunc International).

4. 96-Well collection plates (Nalge Nunc International).

5. $50 \mathrm{~m} M$ Tris- $\mathrm{HCl}$ buffer at $\mathrm{pH} 9.0$ (W1 ion exchange bead washing buffer).

6. $50 \mathrm{~m} M$ Tris- $\mathrm{HCl}$ buffer containing $9 \mathrm{~mol} / \mathrm{L}$ urea and $20 \mathrm{~g} / \mathrm{L}$ CHAPS $(3,3-$ cholmido-propyl-dimethylammonio-1-propanesulfonate) (D1 sample denaturing buffer) at $\mathrm{pH} 9.0$.

7. $50 \mathrm{~m} M$ Tris- $\mathrm{HCl}$ buffer containing $1 \mathrm{~mol} / \mathrm{L}$ urea and $2.2 \mathrm{~g} / \mathrm{L}$ CHAPS at $\mathrm{pH} 9.0$ (Q1 bead equilibration buffer).

8. $50 \mathrm{~m} M$ Tris- $\mathrm{HCl}$ buffer containing $1 \mathrm{~g} / \mathrm{L} N$-octyl- $\beta$-D-glucopyranoside (OGP) at pH 9.0 (E1 elution buffer).

9. $100 \mathrm{~m} M$ Sodium phosphate containing $1 \mathrm{~g} / \mathrm{L}$ OGP at $\mathrm{pH} 7.0$ (E2 elution buffer).

10. $100 \mathrm{~m} M$ Sodium acetate containing $1 \mathrm{~g} / \mathrm{L}$ OGP at $\mathrm{pH} 5.0$ (E3 elution buffer).

11. $100 \mathrm{~m} M$ Sodium acetate containing $1 \mathrm{~g} / \mathrm{L}$ OGP at $\mathrm{pH} 4.0$ (E4 elution buffer).

12. $50 \mathrm{~m} M$ Sodium citrate containing $1 \mathrm{~g} / \mathrm{L}$ OGP at $\mathrm{pH} 3.0$ (E5 elution buffer).

13. $33.3 \%$ Isopropanol, $16.7 \%$ acetonitrile, and $0.1 \%$ trifluoroacetic acid (E6 elution buffer).

14. Micromix 5-01 shaker (Euro/DPC Ltd., Gwynedd, UK).

\subsection{Chip Pretreatment, Sample Binding, and Analysis}

1. Copper (II) immobilized metal affinity capture [IMAC30 Cu(II)] ProteinChip ${ }^{\circledR}$ Array (Ciphergen Biosystems Inc., Fremont, CA).

2. Weak cation-exchange (CM10) ProteinChip Array (Ciphergen Biosystems, Inc.).

3. 96-Well ProteinChip bioprocessor (Ciphergen Biosystems, Inc.).

4. $100 \mathrm{~m} M$ Copper sulfate (CuSO4; Sigma-Adrich, St. Louis, MO).

5. $100 \mathrm{~m} M$ Sodium phosphate containing $0.5 \mathrm{~mol} / \mathrm{L} \mathrm{NaCl}$ (IMAC30 chip binding buffer).

6. $100 \mathrm{~m} M$ Sodium acetate buffer $\mathrm{pH} 4.0$ (serves as both IMAC30 chip neutralizing buffer and CM10 chip binding buffer). 
7. $50 \%$ Sinapinic acid (Ciphergen Biosystems Inc.) in $500 \mathrm{~mL} / \mathrm{L}$ acetonitrile and 5 $\mathrm{mL} / \mathrm{L}$ trifluoroacetic acid (Sigma-Adrich).

8. Protein Biological System (PBS) IIc mass spectrometer reader (Ciphergen Biosystems).

9. All-in-1 peptide molecular mass standard (Ciphergen Biosystems Inc.).

10. Ciphergen ProteinChip Software 3.0.2 (Ciphergen Biosystems Inc.).

\section{Methods}

\subsection{Treatment of SARS Patients}

1. Patients with fever and other symptoms of respiratory infection are initially managed with broad-spectrum antibiotics and supportive therapy.

2. After the diagnosis of clinical SARS is made and if there is no response to antibiotic therapy, combination therapy with ribavirin and systemic steroids is initiated.

3. Intravenous pulse methylprednisolone is initiated when the clinical condition, radiological presentation, or oxygen saturation status of the SARS patients further deteriorates.

4. The clinical characteristics, detailed management plan, and treatment regimen of this cohort of patients can be referred to a previous publication from our hospital (3).

\subsection{Serial Chest Radiographic Score}

The extent of pneumonia in the SARS patients with longitudinal follow-up is assessed by a radiologist according to a serial chest radiographic score, modified from a score system initially proposed to assess computer tomography of the chest (16) and summarized as in Fig. 2.

1. After X-ray chest radiography is taken, divide the frontal chest X-ray radiograph into six lung zones, namely, left upper zone, left middle zone, left lower zone, right upper zone, right middle zone, and right lower zone.

2. The upper zone (left or right) represents area above carina (including the apex).

3. The middle zone (left or right) represents area from carina to the level of inferior pulmonary veins.

4. The lower zone (left or right) represents area from the lower margin of middle zone to the lung base.

5. Score the opacity in each lung zone by a "coarse semiquantitative method" with a five points'scale of grades $0-4$.

6. Grade 0 represents no opacity involved area.

7. Grade 1 represents $5-24 \%$ opacity involved areas.

8. Grade 2 represents $25-49 \%$ opacity involved areas.

9. Grade 3 represents $50-74 \%$ opacity involved areas.

10. Grade 4 represents $75-100 \%$ opacity involved areas.

11. Add the grading from each of the six lung zones to provide a 0-24 point summation scale for assessing the extent of pneumonia. 


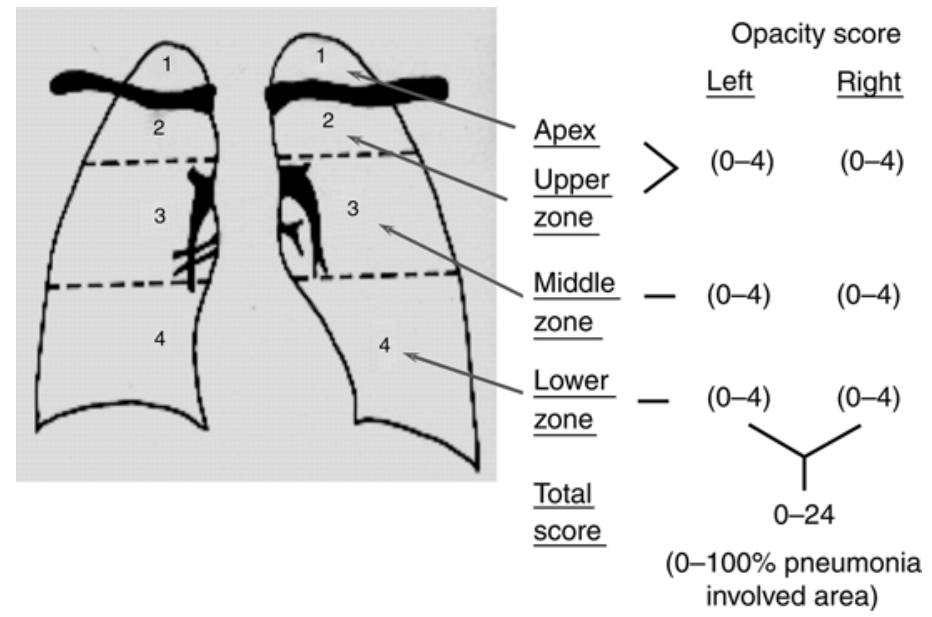

Fig. 2. Assessing the extent of pneumonia by chest X-ray opacity score. The opacity in each zone (apex+upper, middle, and lower zones) from the left and right regions of the lung was scored by a "coarse semiquantitative method" with a 5 points'scale of grades $0-4$ representing involved areas of $0,5-24,25-49,50-74$, and $75-100 \%$, respectively. A total score was then calculated by adding up all the grades in the six zones (Apex and upper zones were considered as one zone) to provide a 0-24 points'scale with the higher number representing more severe pneumonic disease.

\subsection{Serum Preparation}

1. $8 \mathrm{~mL}$ Blood is collected from each patient or control subject.

2. Incubate the blood for approx $2 \mathrm{~h}$ at $4^{\circ} \mathrm{C}$ to clot the blood cells.

3. Separate the serum portion from the clotted cells by centrifugation at $230 \mathrm{~g}$ for $15 \mathrm{~min}$.

4. Aliquot the sera for various routine laboratory diagnostic tests.

5. Freeze the remaining sera at $-70^{\circ} \mathrm{C}$ for protein chip array profiling analysis.

\subsection{Ion Exchange Fractionation of Serum Proteins}

Owing to the protein complexity in the serum sample, an initial fractionation in Q Ceramic Hyper D F ion exchange sorbent beads is performed to generate serum fractions for chip binding experiments (Fig. 1) $(\mathbf{1 0}, 11)$.

\subsubsection{Washing of Ion Exchange Beads}

1. Wash Q Ceramic Hyper D Fanion exchange sorbent beads three times each with five bed volumes of W1 bead washing buffer.

2. Drain the washing buffer each time in vacuum after washing.

3. Keep beads in $50 \%$ suspension in $\mathrm{W} 1$ buffer at RT. 


\subsubsection{Denaturation of Serum Proteins}

1. Thaw serum from $-70^{\circ} \mathrm{C}$ freezer immediately before serum fractionation.

2. Aliquot $20 \mu \mathrm{L}$ of serum to each well in a 96-well sample plate.

3. Add $30 \mu \mathrm{L}$ of $\mathrm{D} 1$ denaturing buffer to the well containing the serum to denature the serum proteins.

4. Shake the sample plate on a Micromix 5-01 shaker vigorously (in an amplitude of 7 and form of $20 \mathrm{Hg}$ ) for $20 \mathrm{~min}$ at $4^{\circ} \mathrm{C}$ to mix the serum with the D1 buffer well.

\subsubsection{Equalibration of Ion Exchange Beads and Binding of Serum Samples}

1. Add $180 \mu \mathrm{L}$ of $50 \%$ suspension of ion exchange beads to each well of a 96 -well Silent Screen filter plate and drain off the W1 bead-washing buffer by vacuum.

2. Wash the ion exchange beads in each well three more times by adding $200 \mu \mathrm{L}$ of Q1 bead equilibration buffer to the beads and draining off the buffer by vacuum.

3. After the last vacuum drain, transfer $50 \mu \mathrm{L}$ of the denatured serum sample from the well in the sample plate to the corresponding well in the filter plate containing the equilibrated ion exchange beads.

4. Add $50 \mu \mathrm{L}$ of $\mathrm{Q} 1$ buffer to the well in the sample plate where serum was originally placed and rinse the residual serum by pipetting up and down five times.

5. Transfer the $50 \mu \mathrm{L}$ of the serum rinse to the well in the filter plate containing the mixture of denatured serum sample and ion exchange beads.

6. Shake the filter plate vigorously on the Micromix shaker (in an amplitude of 7 and form of $20 \mathrm{Hg}$ ) for $30 \mathrm{~min}$ at $4^{\circ} \mathrm{C}$ to mix the serum and beads well.

\subsubsection{Elution of Serum Fractions}

1. After shaking, place a 96-well F1 collection plate under the filter plate and centrifuge at $1600 \mathrm{~g}$ for $1 \mathrm{~min}$ to collect the flow-through fraction in the wells of the F1 collection plate.

2. Add $100 \mu \mathrm{L}$ of E1 elution buffer (at pH 9.0) to each well in the filter plate containing the serum bound beads and shake vigorously again on orbital shaker (speed and time as before) for $10 \mathrm{~min}$ at RT.

3. Collect the eluate in the same collection plate F1 by centrifugation at $1600 \mathrm{~g}$ for 1 min again.

4. This represents fraction 1 , which contains both the flow-through fraction at $\mathrm{pH} 9.0$ and the $\mathrm{pH} 9.0$ eluate.

5. Add $100 \mu \mathrm{L}$ of $\mathrm{E} 2$ elution buffer (at $\mathrm{pH}$ 7.0) to each well in the filter plate and again shake at the same speed and time.

6. Collect the eluate in another new F2 collection plate by centrifugation as before.

7. Add $100 \mu \mathrm{L}$ of E2 elution buffer again to each filter well and elute a second time by shaking and centrifugation as before onto $\mathrm{F} 2$ collection plate again.

8. This represents fraction 2 , which contains the $\mathrm{pH} 7.0$ eluate. 
9. Elute the serum protein bound beads similarly in turn with E3, E4, and E5 elution buffers as above resulting in fractions 3 , 4, and 5 containing $\mathrm{pH} 5.0$, $\mathrm{pH} 4.0$, and $\mathrm{pH} 3.0$ eluates, respectively, in three new F3, F4, and F5 collection plates.

10. Final elution is achieved by adding E6 organic solvent elution buffer and centrifugation at $2000 \mathrm{~g}$ for $5 \mathrm{~min}$ giving rise to fraction 6 containing the organic solvent eluate in collection plate F6.

11. Freeze fractions $1-6$ in collection plates E1-6 at $-70^{\circ} \mathrm{C}$ until the chip binding protocol proceeds.

\subsection{Chip Pretreatment}

The ProteinChip Array Profiling technique is also called surface enhanced laser desorption and ionization time of flight mass spectrometry (SELDI-TOFMS, Ciphergen Biosystems Inc., Fremont, CA). The chip array used is a $10 \mathrm{~mm}$ wide $\times 80 \mathrm{~mm}$ long metal chip having eight $2-\mathrm{mm}$ spots with a specific chromatographic surface for binding of biomarkers of interest (see Fig. 1). For IMAC30 $\mathrm{Cu}$ (II) ProteinChip Array, a pretreatment procedure is required for loading copper ions onto the chip for binding protein biomarkers with affinity to copper ions. This metal loading step is, however, not required for CM10 ProteinChip Array, which is impregnated with carboxylate ions as weak cation exchanger for biomarker binding. CM10 chips only requires buffer washing instead. Both types of chips are tested to be ideal for binding serum proteins/peptides for the study.

\subsubsection{IMAC30 Chip Pretreatment}

1. Assemble the 96-well bioprocessor for chip pretreatment by placing 12 strips of protein chips in the base clamp assembly and then putting a 96-hole rubber gasket sheet and a 96-well bioprocessor reservoir on top of the chips.

2. Clamp this sandwich tight with the 96 incubation wells in the reservoir placed directly on top of the 96 chip spots.

3. Add $50 \mu \mathrm{L}$ of $100 \mathrm{mM}$ CuSO4 solution into each well in the reservoir making direct contact with each IMAC30 chip spot.

4. Get rid of air bubbles if present.

5. Shake the chips in the bioprocessor for $5 \mathrm{~min}$ at the same speed and time as before in the orbital shaker at RT to allow copper ions from $\mathrm{CuSO} 4$ solution to bind to the chip surface.

6. Pipet away the $\mathrm{CuSO} 4$ solution.

7. Rinse the chip spot in each well with $100 \mu \mathrm{L}$ of Milli-Q grade of water and shake again under the same condition for $1 \mathrm{~min}$ at RT.

8. Rinse each well with $100 \mu \mathrm{L}$ of sodium acetate buffer at $\mathrm{pH} 4.0$ (IMAC30 chip neutralizing buffer) for $5 \mathrm{~min}$ with shaking at RT. 
9. Remove the neutralizing buffer after shaking.

10. Rinse the well again with $100 \mu \mathrm{L}$ of Milli-Q water by shaking for $1 \mathrm{~min}$ at room temperature.

11. Add $150 \mu \mathrm{L}$ of $100 \mathrm{~m} M$ sodium phosphate buffer containing $0.5 \mathrm{~mol} / \mathrm{L} \mathrm{NaCl}$ (IMAC30 chip binding buffer) onto each well and shake for $5 \mathrm{~min}$ at RT to wash the chip spot.

12. Remove the buffer after shaking.

13. Wash with the same IMAC30 chip-binding buffer one more time.

14. Remove the buffer after washing.

15. Proceed immediately to Subheading 3.6.1. for sample binding without letting the chip surface to dry.

\subsubsection{CM10 Chip Pretreatment}

1. After assembling the CM10 chips onto the 96-well bioprocessor (see steps 1-2 in Subheading 3.5.1.), add $150 \mu \mathrm{L}$ of $100 \mathrm{~m} M$ sodium acetate buffer at $\mathrm{pH} 4$. (CM10 chip binding buffer) onto each reservoir well and shake vigorously on micromix shaker again as before for $5 \mathrm{~min}$ at RT to wash the chip spot.

2. Remove the buffer from the well.

3. Wash with the same chip-binding buffer one more time.

4. Remove the buffer.

5. Proceed immediately to Subheading 3.6.2. for sample binding without letting the chip surface to dry.

\subsection{Serum Fraction Binding on Chips}

\subsubsection{IMAC30 Chips}

1. Add $80 \mu \mathrm{L}$ of the IMAC30 chip-binding buffer into each well in the bioprocessor set up containing the IMAC30 chips.

2. Add $20 \mu \mathrm{L}$ of each ion exchange bead eluate (serum fractions 1-6) to the well for sample binding.

3. Mix well by shaking for $30 \mathrm{~min}$ at RT.

4. Remove the serum fractions.

5. Wash the chip spot in the well by adding $150 \mu \mathrm{L}$ of IMAC30 chip-binding buffer onto each well and shake for $5 \mathrm{~min}$ at RT.

6. Remove buffer.

7. Repeat washing (steps 5 and $\mathbf{6}$ ) once more.

8. Rinse with water two times by adding $200 \mu \mathrm{L}$ of Milli-Q water to each well and discard immediately.

9. Remove the chips from bioprocessor and air-dry the chips for $5 \mathrm{~min}$.

10. Add $1 \mu \mathrm{L}$ of $50 \%$ sinapinic acid (which is an energy absorbing molecule solution) to each chip spot.

11. Air-dry the chips for about $10 \mathrm{~min}$.

12. Add $1 \mu \mathrm{L}$ of $50 \%$ sinapinic acid and air-dry again.

13. The chip is ready to be read in the PBS IIc mass spectrometer reader. 
14. Remember to process at least one reference control serum concurrently with the patients'samples on each chip for quality control of chip-to-chip variability.

\subsubsection{CM10 Chips}

1. Add $90 \mu \mathrm{L}$ of $100 \mathrm{~m} M$ sodium acetate buffer at $\mathrm{pH} 4.0$ (CM10 chip binding buffer) into each well in the bioprocessor set up containing the CM10 chips.

2. Add $10 \mu \mathrm{L}$ of each ion exchange bead eluate (sample fractions 1-6) to the well for sample binding.

3. Mix well by shaking for $30 \mathrm{~min}$ at RT.

4. Remove the sample fractions.

5. Wash the chip spot in the well by adding $150 \mu \mathrm{L}$ of CM10 chip binding buffer again into each well and shake for $5 \mathrm{~min}$ at RT.

6. Remove buffer.

7. Repeat washing (steps 5 and $\mathbf{6}$ ) once more.

8. Rinse with water two times by adding $200 \mu \mathrm{L}$ of Milli-Q water to each well and discard immediately.

9. Remove the chips from bioprocessor and air-dry the chips for $5 \mathrm{~min}$.

10. Add $1 \mu \mathrm{L}$ of $50 \%$ sinapinic acid to each chip spot.

11. Air-dry the chip for around $10 \mathrm{~min}$.

12. Add $1 \mu \mathrm{L}$ of $50 \%$ sinapinic acid and air-dry again.

13. The chip is ready to be read in the PBS IIc mass spectrometer reader.

14. Process at least one reference control serum concurrently with the patients'samples on each chip for quality control of chip-to-chip variability as before.

\subsection{Chip Reading and Data Acquisition}

The PBS IIc SELDI-TOF mass spectrometer reader is a laser desorption ionization time of flight mass spectrometer equipped with a pulsed ultraviolet nitrogen laser source. When the laser activates the bound serum biomarkers on the chip surface, the biomarkers become desorbed and ionized. Ionized molecules fly along the mass spectrometer to the ion detector in a time of flight manner according to their mass over charge ratio $(\mathrm{m} / \mathrm{z})$. When the ion signal is detected in the ion detector, signal processing is accomplished by high-speed analog-todigital converter linking to a computer. Detected protein biomarkers are displayed in spectral, map or gel view formats by the Ciphergen ProteinChip software 3.0.2. The following steps show how the chips are read in the mass spectrometer.

1. Click the "Sample Exchange Dialog" button from the manu bar of the Ciphergen ProteinChip software 3.0.2 in the PBS IIc SELDI-TOF mass spectrometer reader.

2. Click "Open Lid" button in the "Sample Exchange Dialog" box to open the lid of the chip chamber.

3. Place the sample treated chips into the slot of the chip chamber.

4. Click "Close Lid" button. 


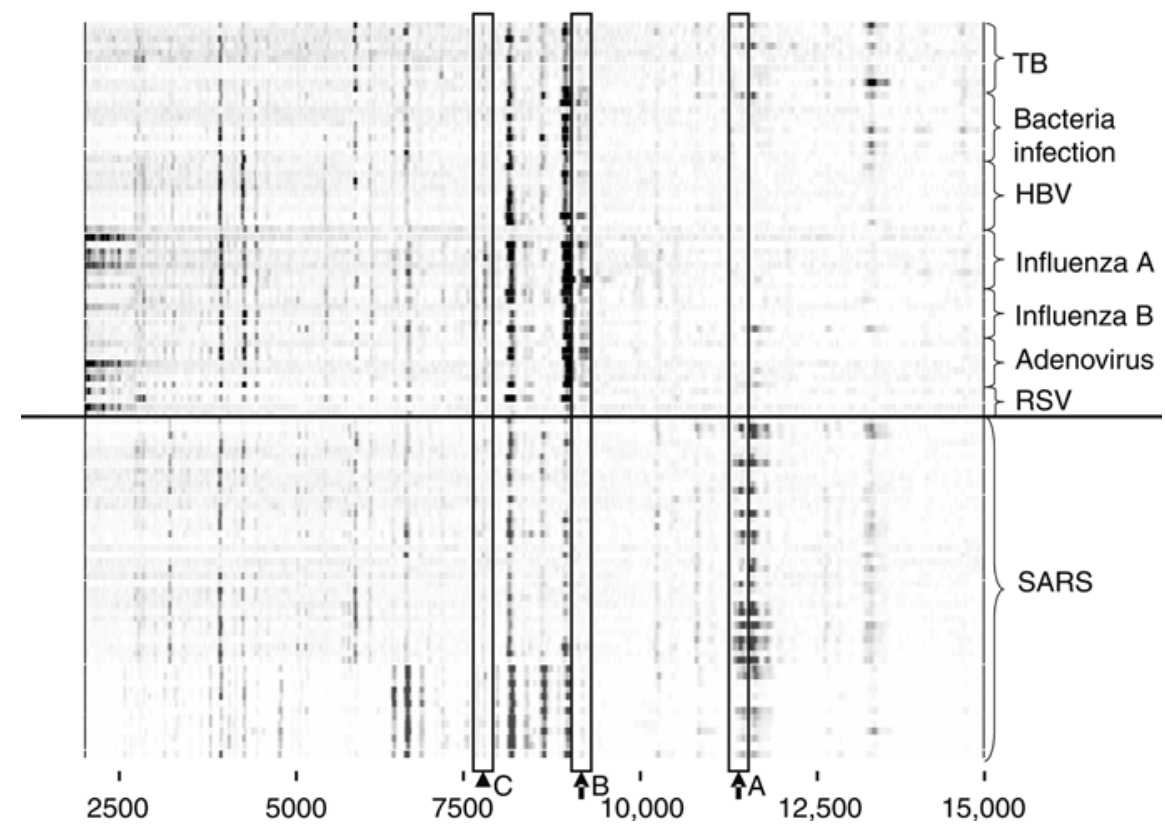

Fig. 3. Gel view of partial protein chip array profiling results in SARS patients'sera vs control infection groups'sera. Part of the profiling results of fraction 1 from the ion exchange serum eluate in SARS patients vs the control patients in $\mathrm{m} / \mathrm{z}$ range from 2000 to 15,000 was illustrated. $\mathrm{HBV}$, hepatitis B virus; TB, M. tuberculosis; RSV, respiratory syncytial virus. The three arrows showed three clusters of biomarkers, $\mathbf{A}-\mathbf{C}$ at $\mathrm{m} / \mathrm{z}$ of 11695, 9159, and 7784, respectively, with cluster $\mathbf{A}$ being significantly increased in SARS patients and the last two clusters $(\mathbf{B}$ and $\mathbf{C})$ significantly decreased.

5. The chip is automatically inserted into the chip chamber.

6. In the "Sample Exchange Dialog" box, input the serial number, chip type, and chip format.

7. Click "Chip" button from the "Sample Exchange Dialog" box.

8. Input sample name and sample group.

9. Click "OK" button.

10. Click "Chip Protocol" button from the menu bar.

11. Input spectrum tag (which is the sample name) and the following spot protocols:

a. Starting laser intensity to 165 .

b. Starting detector sensitivity to 9 .

c. Highest mass of detection to $200,000 \mathrm{Da}$.

d. Optimal mass range of detection from $\mathrm{m} / \mathrm{z}$ of 2000 to 20,000 (signals from $\mathrm{m} / \mathrm{z}$ of 0-2000 are not analyzed as artifacts can be produced by energy-absorbing molecules or other chemical contaminants at this mass range). 


\section{Table 1}

\section{Serum Biomarkers Significantly Increased or Decreased in SARS Patients vs Seven Control Groups of Patients With Other Infections and Healthy Individuals}

\begin{tabular}{lclr}
\hline $\begin{array}{l}\text { Biomarker } \\
\text { number }\end{array}$ & $\begin{array}{c}\text { Mass over } \\
\text { charge }(\mathrm{m} / \mathrm{z})\end{array}$ & $\begin{array}{l}\text { Differential } \\
\text { expression }\end{array}$ & $p$-value ${ }^{a}$ \\
\hline 1 & 4922 & Increased & $1.1 \times 10^{-4}$ \\
2 & 5104 & Increased & $7.1 \times 10^{-8}$ \\
3 & 5215 & Increased & $6.4 \times 10^{-4}$ \\
4 & 5833 & Increased & $3.1 \times 10^{-9}$ \\
5 & 7784 & Decreased & $4.9 \times 10^{-8}$ \\
6 & 8416 & Decreased & $1.6 \times 10^{-7}$ \\
7 & 9159 & Decreased & $1 \times 10^{-10}$ \\
8 & 10,867 & Increased & $3.6 \times 10^{-7}$ \\
9 & 11,508 & Increased & $1.3 \times 10^{-5}$ \\
10 & 11,695 & Increased & $5.4 \times 10^{-6}$ \\
11 & 11,871 & Increased & $8.4 \times 10^{-4}$ \\
12 & 14,715 & Increased & $3.9 \times 10^{-9}$ \\
\hline
\end{tabular}

${ }^{a} p$-values were obtained in Mann-Whitney $U$-test by comparing the normalized peak intensities of the biomarkers in 44 sera from 24 SARS patients vs those in 72 sera from 51 control patients with various viral or bacterial infections (see Materials for individual control group) plus 10 sera from 10 apparently healthy individuals.

e. Focus lag time at $800 \mathrm{~ns}$.

f. Mass deflector to Auto.

g. Data acquisition method to SELDI quantitation.

h. 338 laser shots per sample.

12. Click "Start Running" button from the menu bar.

13. By the time 338 laser shots are fired, the collected data is automatically saved.

14. It is important to first carry out external calibration of the equipment using the AllIn-1 Peptide molecular mass standard (according to the instruction sheet from the manufacturer) to ensure mass accuracy of the spectra before running the samples.

15. Carry out baseline subtraction and calibration for the spectra.

16. Generate labeled peak groups (clusters) across multiple spectra by the Biomarker Wizard mode.

17. Compare peak groups detected in the SARS patients with those of the controls by nonparametric two sample Mann-Whitney $U$-test in the Biomarker Wizard mode.

18. Biomarker Wizard operates in two passes, with the first pass uses low sensitivity settings to detect obvious and well-defined peaks and the second pass uses higher sensitivity settings to search for smaller peaks.

19. This will identify both strong and weak peaks that are significantly increased or decreased in the patients vs the controls. 


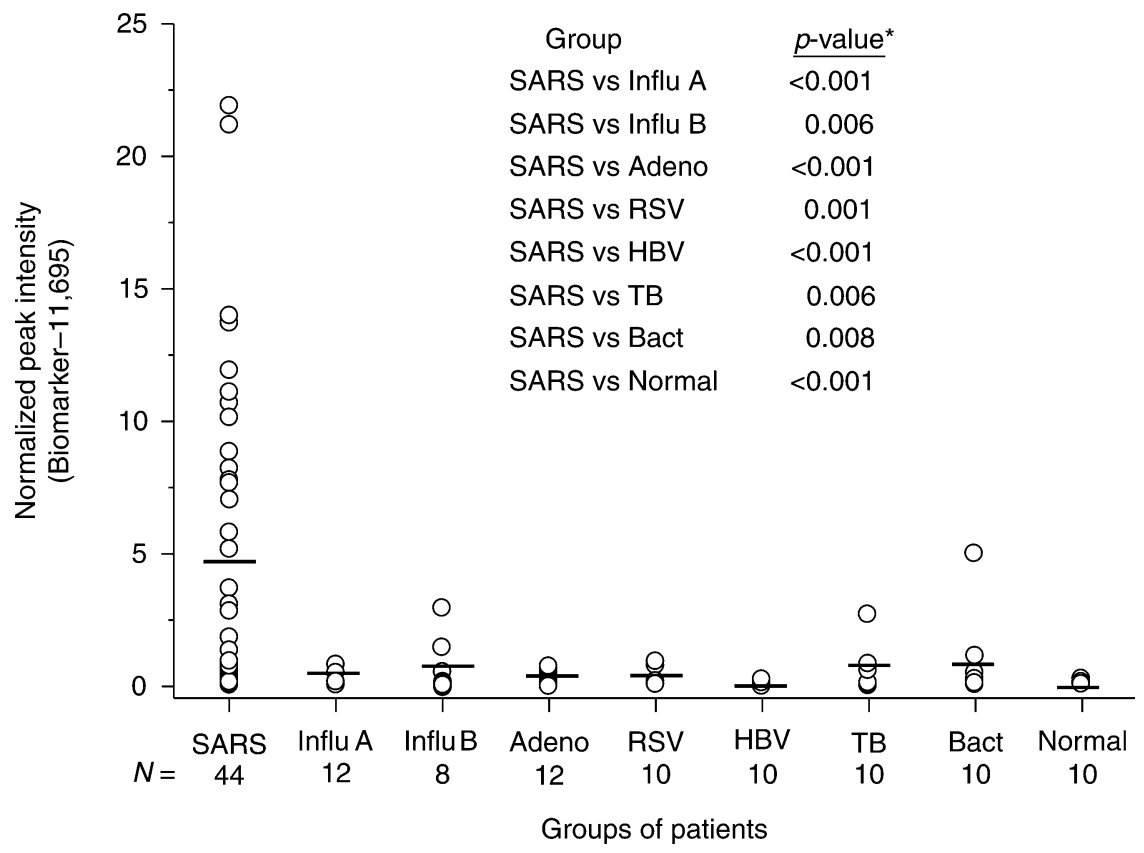

Fig. 4. Comparison of normalized peak intensities of the biomarker at $\mathrm{m} / \mathrm{z}$ of 11,695 in sera from SARS patients and control groups. The normalized peak intensities of this marker in SARS patients'sera were compared with those of the other seven control infection groups and the normal individuals by Mann-Whitney $U$-test. A $p$-value of 0.05 or less demonstrated a statistically significant difference between the two groups. Adeno, adenovirus; Bact, bacterial culture positive; HBV, hepatitis B virus; Influ A, influenza A virus; Influ B, influenza B virus; Normal, apparently normal subjects; RSV, respiratory syncytial virus; TB, M. tuberculosis.

\subsection{Data Analyses}

1. As an example, Fig. 3 illustrates a comparison of multiple biomarkers in serum fraction 1 ( $\mathrm{pH} 9.0$ eluate) of SARS patients vs all the other controls in a gel view format in the $\mathrm{m} / \mathrm{z}$ range of $2000-15,000$.

2. Highlighted in three boxes (A, B, and C) with arrows are biomarkers at $\mathrm{m} / \mathrm{z}$ of $11,695,9159$, and 7784 , respectively with biomarker at 11,695 being significantly increased in the SARS patients vs the controls and biomarkers 9159 and 7784 being significantly decreased.

3. Nine biomarker were found within $\mathrm{m} / \mathrm{z}$ range of 4900-15,000 with highly significant increase in their normalized peak intensities in the SARS patients vs those of the controls and three biomarkers with highly significant decrease (Table 1). 


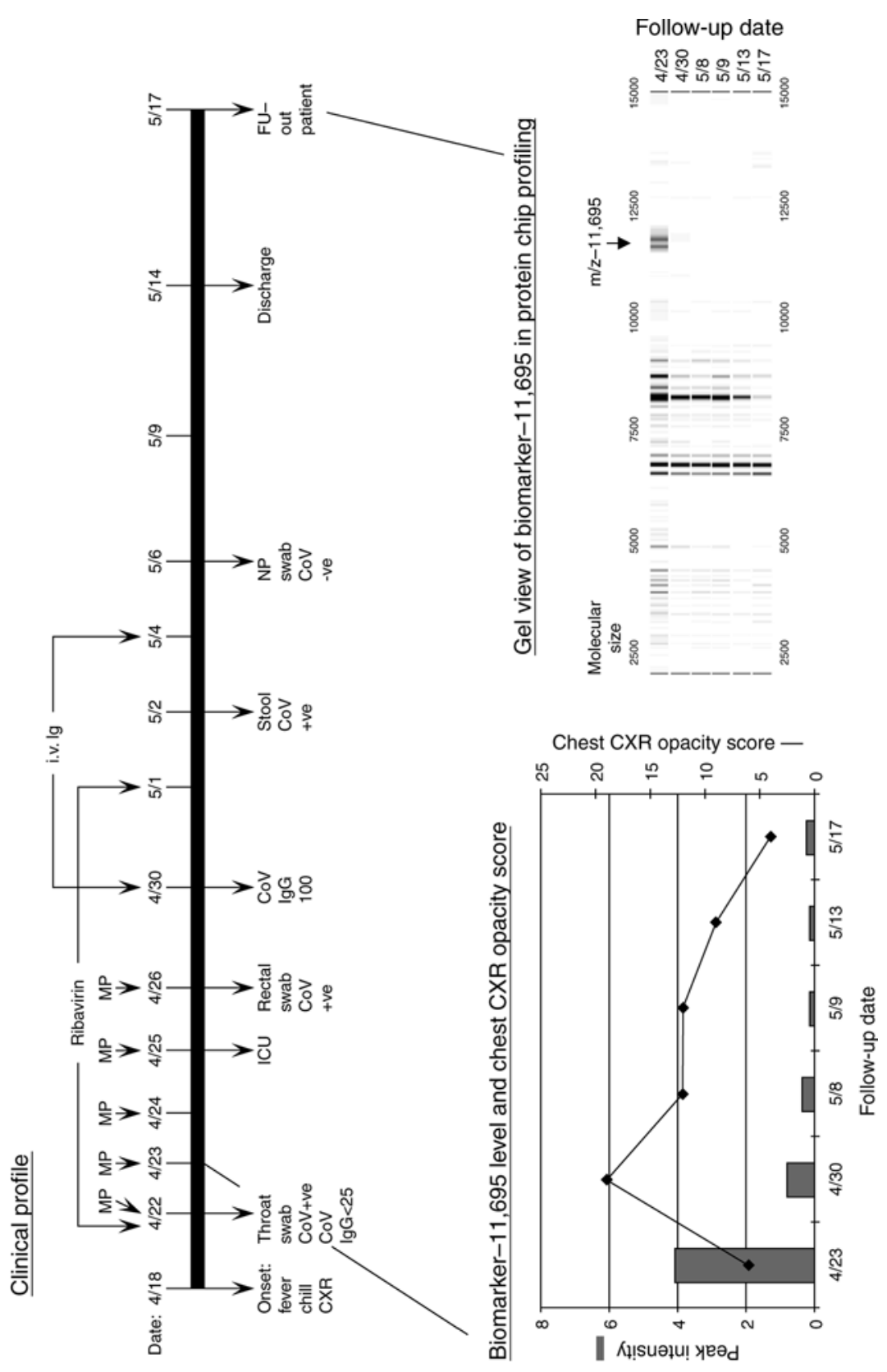


4. Separating the controls into individual groups, a highly significant increase of biomarker at 11,695 is also observed in SARS patients vs each control group (Fig. 4).

5. As pneumonia is a frequent life threatening clinical manifestation in the SARS patients, we further monitor the biomarker-11,695 level in a SARS patient longitudinally (Fig. 5) and correlate the biomarker level with the extent of pneumonia by chest X-ray opacity score (Fig. 2 ).

6. Figure 5 illustrates, in this patient, an elevation of the biomarker-11,695 level preceding the development of pneumonia at the onset of disease (as indicated by an increasing opacity score) followed by a rapid drop of the biomarker level down to the background on recovery from the illness after stringent antiviral and steroid treatments (see Note 1 for details of clinical presentation of the patients).

7. On the other hand, Fig. 6 shows low biomarker-11,695 level during the whole monitoring period in another SARS patient who has low X-ray scores and whose clinical course is relatively mild (see Note 2).

8. By means of peptide mapping and tandem MS/MS mass spectrometry, this biomarker is identified to be Serum Amyloid A protein (SAA), which is an acute phase reactant frequently and rapidly induced in abundance at pneumonia (see ref. 15 for the protein ID results and ref. 17-20 for the techniques adopted).

9. This serial study demonstrates the potential of the biomarkers discovered by protein chip array profiling in monitoring the disease manifestation in SARS patients.

\section{Notes}

1. Clinical and protein chip monitoring of the first SARS patient (see Fig. 5): one day after SARS-CoV infection was diagnosed, the radiographic score of this patient was increased from 6 to a peak value of $>16$ and then dropped to 12,9 , and finally

Fig. 5. (Opposite page) Correlation of the level of serum biomarker-11,695 with the extent of pneumonia in a SARS patient under clinical follow-up. Longitudinal follow-up of the clinical profile of this patient was illustrated in the top panel. Monitoring of the biomarker-11,695 level and chest X-ray opacity score (an indicator of the extent o pneumonia) was shown in the lower left panel. The lower right panel illustrates the biomarker-11,695 in a gel view measured at different time points. CoV IgG $<25$ and 100, Serum SARS-Coronavirus IgG antibody titers of $<1 / 25$ and 1/100; CXR, Lung consolidation as shown by chest X-ray imaging; FU outpatient, During follow-up as outpatient; ICU, Admitted to Intensive care unit; i.v. Ig, Intravenous injection of immune globulin; MP, Daily treatment by $500 \mathrm{mg} \times 2$ of methylprednisolone; NP Swab CoV -ve, RT-PCR negative for SARS Coronavirus in nasopharyngeal swab; Rectal Swab CoV +ve, RT-PCR positive for SARS Coronavirus in rectal swab; Ribavirin, Daily treatment by $400 \mathrm{mg}$ Ribavirin; Stool CoV +ve, RT-PCR positive for SARS Coronavirus in the stool; Throat $\mathrm{Swab} \mathrm{CoV}+\mathrm{ve}$, RT PCR positive for SARS Coronavirus in throat swab. 


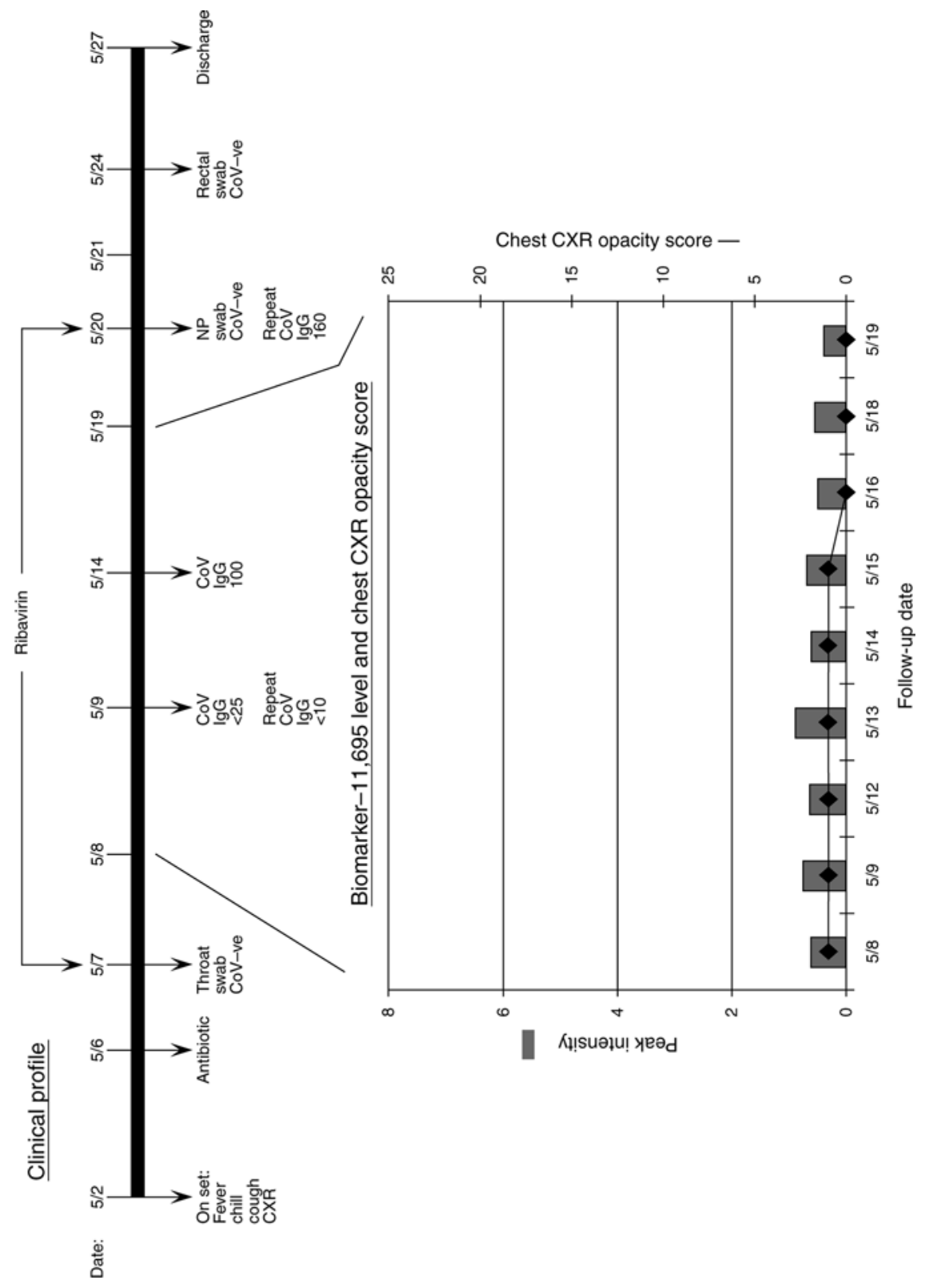


to 4 demonstrating a progressive recovery. The biomarker-11,695 level by protein chip array profiling study showed an elevation that peaked earlier than the radiographic score but it then gradually subsided along with the score to a nadir when the patient was discharged.

2. Clinical and protein chip monitoring of the second SARS patient (see Fig. 6): the second patient had all the typical clinical symptoms of SARS-CoV infection with left upper lobe consolidation in chest radiographs. Despite negative SARS-CoV RT-PCR, titer elevation in paired anti-SARS CoV serum antibody tests performed in two laboratories confirmed the viral infection. He was managed as SARS on the next day. The clinical course was uneventful and uncomplicated. His pneumonia had never been extensive and hence his radiographic score showed just a low level of lung involvement from May 8th to 15th and then totally subsided from May 16th onwards. Biomarker-11,695 level was essentially low throughout the monitoring period.

\section{Acknowledgments}

The authors thank our Kowloon Central Cluster/Queen Elizabeth Hospital Chief Executive, Dr. C. T. Hung and the SARS Management Committee for their support in this project. We also express our appreciation to Dr. Dominic N. C. Tsang, Dr. John K. C. Chan, Dr. King-Chung Lee, Dr. Angel Chan, Dr. Cesar S. C. Wong, Mr. K. H. Leung, Mr. C. C. Tiu, Mr. Gene G. T. Lau, and Miss Elena S. F. Lo in the Pathology Department of Queen Elizabeth Hospital for the provision of SARS patients' sera and their help in this project. The authors thank Dr. Wilina W. L. Lim for the provision of control sera from patients with upper respiratory infection and Dr. Ting-Lok Kwan for the provision of the serial chest X-ray score. We express our gratitude to Mr. Cadmon K. P. Lim in his technical assistance in this project. This work was supported in part by grants from Queen Elizabeth Hospital and Hong Kong Jockey Club. Part of the contents in this book chapter has been reproduced with permission from Yip et al., 2005 see ref. 15 (Copyright 2004 AACC).

Fig. 6. (Opposite page) Correlation of the level of serum biomarker-11,695 with the extent of pneumonia in the second SARS patient under clinical follow-up. Longitudinal follow-up of the clinical profile of this patient was illustrated in the top panel. Monitoring of the biomarker-11,695 level and chest X-ray opacity score was shown in the lower panel. Annotations were similar to those as tabulated in Fig. 5. Antibiotic, Treatment with conventional antibiotics; CoV IgG $<10$ and 160, Serum SARS-Coronavirus IgG antibody titers of $<1 / 10$ and 1/160; NP Swab CoV -ve, RTPCR negative for SARS Coronavirus in nasopharyngeal swab; Rectal Swab CoV -ve, RT-PCR negative for SARS Coronavirus in rectal swab; Throat Swab CoV -ve, RT PCR negative for SARS Coronavirus in throat swab. 


\section{References}

1. World Health Organization. (2003) WHO issued consensus document on the epidemiology of SARS. WHO Wkly Epidemiol. Rec. 78, 373-375.

2. Engel, J. P. (1995) Viral upper respiratory infections. Semin. Respir. Infect. 10, 3-13.

3. Chan, J. W. M., Ng, C. K., Chan, Y. H., et al. (2003) Short term outcome and risk factors for adverse clinical outcomes in adults with severe acute respiratory syndrome (SARS). Thorax 58, 686-689.

4. Donnelly, C. A., Ghani, A. C., Leung, G. M., et al. (2003) Epidemiological determinants of spread of casual agent of severe acute respiratory syndrome in Hong Kong. Lancet 361, 1761-1766.

5. Yam, W. C., Chan, K. H., Poon, L. L., et al. (2003) Evaluation of reverse transcriptase-PCR assays for rapid diagnosis of severe acute respiratory syndrome associated with a novel coronavirus. J. Clin. Microbiol. 41, 4521-4524.

6. Xu, G., Lu, H., Li, J., et al. (2003) Primary investigation on the changing mode of plasma specific IgG antibody in SARS patients and their physicians and nurses. Beijing Da Xие Xие Bao 35, 23-25.

7. Poon, L. L. M., Wong, O. K., Luk, W., Yuen, K. Y., Peiris, J. S. M., and Guan, Y. (2003) Rapid diagnosis of a coronavirus associated with severe acute respiratory syndrome (SARS). Clin. Chem. 49, 953-955.

8. Grinblat, L., Shulman, H., Glickman, A., Matukas, L., and Paul, N. (2003) Severe acute respiratory syndrome: radiographic review of 40 probable cases in Toronto, Canada. Radiology 228, 802-809.

9. Muller, N. L., Ooi, G. C., Khong, P. L., and Nicolaou, S. (2003) Severe acute respiratory syndrome: radiographic and CT findings. AJR Am. J. Roentgenol. 181, 3-8.

10. Fung, E. T., Thulasiraman, V., Weinberger, S. R., and Dalmasso, E. A. (2001) Protein biochips for differential profiling. Curr. Opin. Biotechnol. 12, 65-69.

11. Wright, G. L. Jr. (2002) SELDI proteinchip MS: a platform for biomarker discovery and cancer diagnosis. Expert Rev. Mol. Diagn. 2, 549-563.

12. Weinberger, S. R., Dalmasso, E. A., and Fung, E. T. (2002) Current achievements using ProteinChip Array technology. Curr. Opin. Chem. Biol. 6, 86-91.

13. Issaq, H. J., Veenstra, T. D., Conrads, T. P., and Felschow, D. (2002) The SELDITOF MS approach to proteomics: protein profiling and biomarker identification. Biochem. Biophys. Res. Commun. 292, 587-592.

14. Vorderwulbecke, S., Cleverley, S., Weinberger, S. R., and Wiesner, A. (2005) Protein quantification by the SELDI-TOF-MS-based ProteinChip ${ }^{\circledR}$ system. Nat. Method 2, 393-395.

15. Yip, T. T. C., Chan, J. W. M., Cho, W. C. S., et al. (2005) protein chip array profiling analysis in patients with severe acute respiratory syndrome identified serum amyloid a protein as a biomarker potentially useful in monitoring the extent of pneumonia. Clin. Chem. 51, 47-55.

16. Ng, C. S., Desai, S. R., Rubens, M. B., Padley, S. P. G., Wells, A. U., and Hansell, D. M. (1999) Visual quantitation and observer variation of signs of small airways disease at inspiratory and expiratory CT. J. Thorac. Imag. 14, 279-285. 
17. Cleveland, D. W., Fischer, S. G., Kirschner, M. W., and Laemmli, U. K. (1977) Peptide mapping by limited proteolysis in sodium dodecyl sulfate and analysis by gel electrophoresis. J. Biol. Chem. 252, 1102-1106.

18. Baudys, M., Foundling, S., Pavlik, M., Blundell, T., and Kostka, V. (1988) Protein chemical characterization of Mucor pusillus aspartic proteinase amino acid sequence homology with the other aspartic proteinases disulfide bond arrangement and site of carbohydrate attachment. FEBS Lett. 235, 271-274.

19. Fournier, I., Chaurand, P., Bolbach, G., Lutzenkirchen, F., Spengler, B., and Tabet, J. C. (2000) Sequencing of a branched peptide using matrix-assisted laser desorption/ionization time-of-flight mass spectrometry. J. Mass Spectrom. 35, $1425-1433$.

20. Perkins, D. N., Pappin, D. J., Creasy, D. M., and Cottrell, J. S. (1999) Probabilitybased protein identification by searching sequence databases using mass spectrometry data. Electrophoresis 20, 3551-3567. 